\title{
THE
}

\section{The Evolution of Difficult Childbirth and Helpless Hominin Infants}

Holly M. Dunsworth

University of Rhode Island, holly_dunsworth@uri.edu

Leah Eccleston

University of Rhode Island

Follow this and additional works at: https://digitalcommons.uri.edu/soc_facpubs

The University of Rhode Island Faculty have made this article openly available. Please let us know how Open Access to this research benefits you.

This is a pre-publication author manuscript of the final, published article.

Terms of Use

This article is made available under the terms and conditions applicable towards Open Access Policy Articles, as set forth in our Terms of Use.

\section{Citation/Publisher Attribution}

Dunsworth, Holly and Leah Eccleston. The Evolution of Difficult Childbirth and Helpless Hominin Infants. Annual Review of Anthropology, vol. 44, no. 1, 2015, pp. 55-69. http://dx.doi.org/10.1146/annurevanthro-102214-013918

Available at: http://dx.doi.org/10.1146/annurev-anthro-102214-013918

This Article is brought to you for free and open access by the Sociology \& Anthropology at DigitalCommons@URI. It has been accepted for inclusion in Sociology \& Anthropology Faculty Publications by an authorized administrator of DigitalCommons@URI. For more information, please contact digitalcommons-group@uri.edu. 


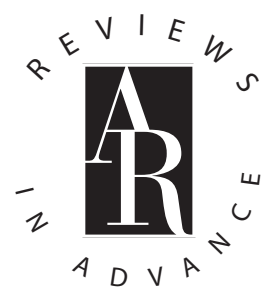

\section{The Evolution of Difficult Childbirth and Helpless Hominin Infants}

\section{Holly Dunsworth and Leah Eccleston}

Department of Sociology and Anthropology, University of Rhode Island, Kingston, Rhode Island 02881; email: holly_dunsworth@uri.edu, leah_eccleston@my.uri.edu
Annu. Rev. Anthropol. 2015. 44:55-69

The Annual Review of Anthropology is online at anthro.annualreviews.org

This article's doi:

10.1146/annurev-anthro-102214-013918

Copyright (c) 2015 by Annual Reviews. All rights reserved

\section{Keywords}

gestation, obstetric dilemma, obstetrical dilemma, paleoanthropology, parturition, pregnancy

\begin{abstract}
Because of the implications for behavioral, social, and cultural evolution, reconstructions of the evolutionary history of human parturition are driven by two main questions: First, when did childbirth become difficult? And second, does difficult childbirth have something to do with infant helplessness? Here we review the available evidence and consider answers to these questions. Although the definitive timeframe remains unclear, childbirth may not have reached our present state of difficulty until fairly recently $(<500,000$ years ago) when body and brain sizes approximated what we have now, or perhaps not until even more recently because of agriculture's direct and indirect effects on the growth and development of both mother and fetus. At present, there is little evidence to indicate that difficult childbirth has affected the evolution of gestation length or fetal growth, selecting for infants that are born in a supposed underdeveloped state, although these phenomena likely share causes.
\end{abstract}




\section{INTRODUCTION}

Bearing and rearing human infants involve processes that appear to be different from those that perpetuate other lineages. Among primates and across mammals, humans seem to have exceptionally protracted and painful labor, with a surprisingly high injury and failure rate, and remarkably helpless babies. Furthermore, a woman's pregnancy, labor, parturition, and infant care are nearly always assisted, suggesting that cooperative breeding (Clutton-Brock 2002; Kramer 2005; Hrdy 2009) is adaptive in the human species because of its direct contribution to the reproductive success of the maternal and paternal lines and its major contribution to that of the offspring.

Reconstructing the origins and evolution of childbirth difficulty, as well as its consequences, is an important anthropological enterprise because $(a)$ childbirth determines whether a lineage continues to evolve; (b) it has important implications for anatomical, behavioral, social, and cultural evolution; and $(c)$ researchers have been increasingly interested in applying an evolutionary perspective to current childbirth practices. Here we review what is known about hominin parturition, with a perspective that looks beyond the fossil record. The discussion is focused on answering two main questions that continue to drive research.

\section{WHEN DID CHILDBIRTH BECOME DIFFICULT?}

Human labor is long, difficult, and painful and is burdened by a notable risk of trauma and mortality to mother and infant (Dolea \& AbouZahr 2003, WHO 2005). It is an extraordinary event among sexually reproducing organisms. To determine when childbirth became difficult, we should first consider the causes. These include a number of factors; however, the causes related to bony pelvic anatomy and other skeletal traits have received much attention from anthropologists because they are applicable to fossil analysis.

In association with and, perhaps, in adaptive response to selection for bipedal posture and gait, the hominin pelvis metamorphosed in ways that included changes to the bony birth canal (Lovejoy 2005). The overall shape of the hominin pelvis became more basin-like-moving the action of the minor gluteals from the back to the sides, changing their role to support the trunk and body during the single-leg-support phase of walking and running. In concert with these changes, the overall shape of the birth canal transformed from an anteroposteriorly elongated opening to an anteroposteriorly short and mediolaterally oblong one (Schultz 1949, Rosenberg \& Trevathan 1995). The pelvic passage, as it exists now, is defined by three planes that differ in shape and orientation. That is, the first aspect that the fetus encounters, the pelvic inlet, is widest in the mediolateral dimension. Next, the midplane is often the narrowest of the three planes. And finally the outlet is widest in the anteroposterior dimension, perpendicular to the shape of the inlet. Thus, for a large portion, perhaps the majority, of human births, the fetus twists while passing through the birth canal during the final stages of labor as its head and its shoulders navigate these dimensions of varying size and shape to exit the mother's body (Abitol 1993, Rosenberg \& Trevathan 2002, Trevathan 2015).

Humans and extinct hominins, with the pelvic indicators of this fetal rotation (or this "birth mechanism" as it is often called; Rosenberg \& Trevathan 2014), appear to require assistance during childbirth (Rosenberg \& Trevathan 2002). Because the baby emerges facing away from the mother, with its occiput anterior, it is purported to be difficult to pull safely out and up to the breast. And, in this orientation, it is difficult for the mother to reach down to clear the mucous away from the baby's airway so that it can safely endure the entire labor. This fetal rotation caused by the human pelvic morphology is considered to be a major contributor to childbirth difficulty. So the reconstruction of the evolution of the hominin birth canal has been an exercise in tracing childbirth difficulty. 
Unfortunately, the fossil record for hominin pelves is smaller than we would prefer. There are few fossil pelvic remains of hominin species that precede those of human-like Neanderthals and anatomically modern Homo sapiens (Trinkaus 1984, Arsuaga et al. 1999). There are 20 total pelvic specimens for Homo erectus and earlier hominins, most of which are fragments that have not been useful for answering questions of childbirth evolution. The few pelves that are relatively well-preserved are incomplete and require reconstruction, which is not performed in exactly the same way by all researchers. Some pelves have disputed sex and/or taxonomic identities (e.g., BSN49/P27, a possible H. erectus from Gona, Ethiopia; Simpson et al. 2008, Ruff 2010). The individuals are also separated by thousands of miles and millions of years, and a key $H$. erectus specimen is an immature male (the "Nariokotome boy" KNM-WT 15000).

It is not surprising that analyses of these few fossil hominin pelves have not converged on a consensus about the origin or evolution of fetal rotation throughout the hominin clade (Weaver \& Hublin 2009, Chene et al. 2014, and others). Fetal rotation in australopiths is disputed. Reconstructions and analyses of the pelves of AL 288-1 ("Lucy" from Hadar, Ethiopia) and STS 14 (from Sterkfontein, South Africa) indicate to some that the inlet and outlet are both widest in the mediolateral dimension; therefore, fetal rotation did not occur in australopiths (Tague \& Lovejoy 1986). Others, however, infer more humanlike rotational mechanics (Berge et al. 1984, Berge \& Goularas 2010). Whether such a phenomenon occurred in earlier hominins such as Ardipithecus (Lovejoy et al. 2009) remains unknown. It is possible that a humanlike pattern of fetal rotation began in $H$. erectus (Chene et al. 2014), but the trait was not necessarily retained in some Neanderthals (Weaver \& Hublin 2009).

Owing to the incomplete fossil record, the evolution of fetal rotation continues to elude paleoanthropologists. However, as increasing instances of nonhuman primate births are observed, the rotational birth process loses significance. That is, nonhuman primates are now known to be born oriented with the offspring's head facing occiput anterior as in human births (Hirata et al. 2011, Trevathan 2015). Regardless, fetal rotation contributes to prolonged and painful labor in humans, especially when combined with the tight fit between the fetus and the birth canal. So if the hominin pelvis causes present fetal rotation, does it also cause the present tight fit? And if so, could we trace the tight fit through the hominin fossil record?

That human pelves are sexually dimorphic in the dimensions that comprise the birth canal is evidence that selection has favored adequate capacity for reproduction (LaVelle 1995, Simpson et al. 2008, Kurki 2013). But the well-known obstetric dilemma (OD) hypothesis posits that selection for bipedalism has imposed a limit on the dimensions of the birth canal and that extant hominins have reached it. That is, selection ramped up hominin neonatal brain size butwas simultaneously prohibited from easing childbirth and lowering trauma and mortality risks 2 use increasing the size of the birth canal would negatively impact bipedalism (e.8., Schultz 1949, Krogman 1951, Washburn 1960, Leutenegger 1982, Trinkaus 1984, Rosenberg 1992, Rosenberg \& Trevathan 1995, Dunsworth et al. 2012, Wells et al. 2012, Roberts \& Thorpe 2014). In this article and elsewhere the OD refers to a human evolutionary hypothesis. However, "obstetric dilemma" is also used by anthropologists as shorthand for the tight fit between pelvis and neonate at birth in extinct and extant humans and in other primates, regardless of the evolutionary explanation (e.g., Wells et al. 2012).

From the OD hypothesis perspective, researchers assume that the greater the biacetabular distance (between the hip joints), the more work that is required of the hip abductors during bipedalism, and therefore the higher the cost and/or the lower the efficiency. A notable drawback to the OD is that although the biomechanical theory is sound, it has not been borne out by studies of human performance. Neither wide hips nor female hips have consistently predicted locomotor 
Macaca

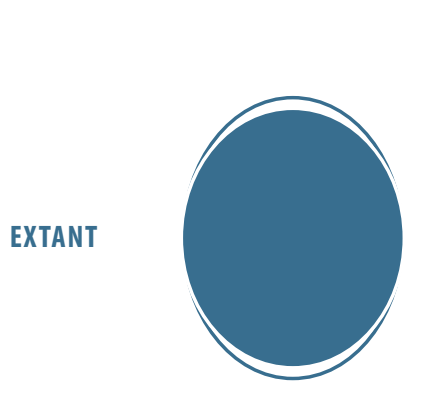

AL 288 -1 (Lucy)

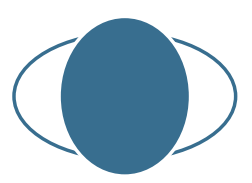

FOSSIL

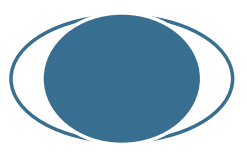

Hylobates

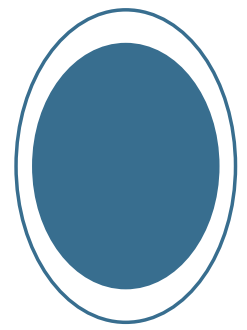

STS 14
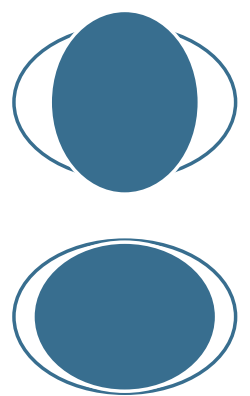

Pan

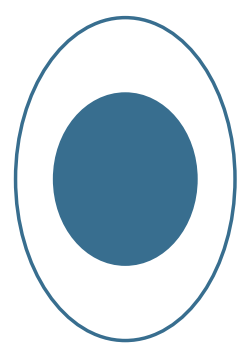

$\mathrm{MH} 2$
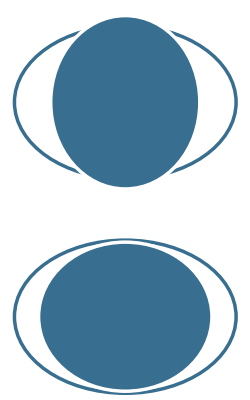

Homo sapiens

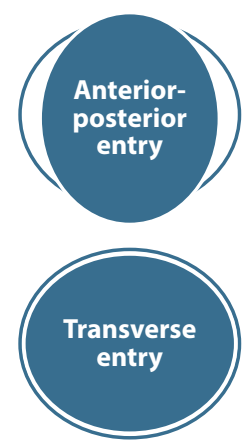

Early Homo (Gona)

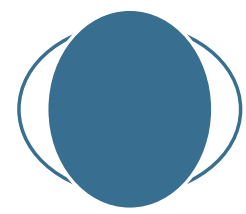

Anteriorposterior entry

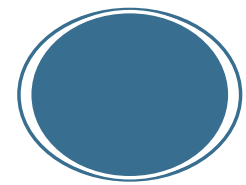

Transverse entry

Figure 1

Tightness of fit at birth is not unique in humans and may precede the modern human postcranium. Filled ovals represent infant crania; outer ovals are maternal pelvic inlet dimensions, which are scaled so that mediolateral dimensions are equal. Representations on top show variation in the tightness of fit in a monkey (Macaca), a gibbon (Hylobates), a chimpanzee (Pan), and humans. The four hominin fossils on the bottom illustrate estimations of the tight fit in Australopithecus (AL 288-1, STS 14, MH 2) and early Homo (Gona). Adapted with permission from Wells et al. (2012).

economy or efficiency (demonstrated and reviewed in Warrener et al. 2015; see also Dunsworth et al. 2012).

Regardless of whether selection for bipedalism (or some other unidentified reason) is limiting the expansion of the birth canal, other nonpelvic traits are contributing to the tight fit: Humans have relatively large and fat babies with large heads and broad shoulders that approach the capacity of the bony birth canal (Schultz 1949, Rosenberg \& Trevathan 2002, Wells et al. 2012; Figure 1). The human condition at birth starkly contrasts that of the other hominoids.

When the mother's body size is taken into account, humans have relatively large neonates with relatively large heads compared with other apes and other primates (Dunsworth et al. 2012). Surely the high percentage of fetal body fat accumulated in the latter stages of gestation contributes to neonatal size (Kuzawa 1998, Aiello \& Wells 2002, Cunnane \& Crawford 2003). And although hominin adiposity likely increased with encephalization, beginning most notably with $\mathrm{H}$. erectus, it is possible that large neonatal size began even earlier in hominin history than the genus Homo.

DeSilva (2011) recently made a rigorous attempt to estimate neonatal size across hominin evolutionary history (Figure 1). By this estimation, it looks like australopiths, with their very small increase in relative brain size, would have had slightly larger neonates for their body sizes. Unfortunately, without fossilized mother-infant dyads, comparing unrelated fossil hominin 
individuals with related pairs of living humans will remain a challenge for constructing models such as DeSilva's. In addition, the contribution of large neonatal size to childbirth difficulty still depends on pelvic dimensions, which for australopiths (listed above) are known only for two incomplete specimens. However, it does raise the possibility that the tight fit and, hence, difficult childbirth arose prior to the modern bipedal skeleton and marked encephalization.

Encephalization likely increased the selection pressure on the size of the hominin birth canal (Abitol 1987), but whether the small brains of australopiths drove this pressure is unclear. Further complicating matters, the partial pelvis of the relatively new species $A$. sediba has been described as having morphological traits like those suggested to be adapted in Homo for birthing encephalized infants (Kibii et al. 2011). So it is possible that the capacity to birth large or large-brained infants arose before the large or large-brained infants did-a scenario that sounds unremarkable given it is what chimpanzees would experience if they were to become more encephalized or larger, overall, at birth. Only more fossils will speak to this potential plot twist in the evolutionary history of childbirth.

In addition to large overall size and brain size, the shoulders also contribute to the tight fit in humans (Trevathan \& Rosenberg 2000, Rosenberg \& Trevathan 2002) and possibly affect the birthing process in apes (Hirata et al. 2011). However, it is possible that human shoulders contribute uniquely to the tight fit, and if so, then fetal australopith shoulders may not have caused a problem. The well-preserved scapulae of the Dikika infant show a cranial orientation of the glenoid fossa (shoulder joint) as opposed to a human-like lateral one, indicating that the shoulders of australopiths were more ape-like (Green \& Alemseged 2012) and may have passed more easily during parturition than do human shoulders.

The delay in infant cranial sutures may have evolved to deliver large-brained babies. Soft neonatal heads are suggested to have increased in prevalence as early as Australopithecus africanus (Falk et al. 2012). However, separate interpretations contest this analysis of the fossil evidence (Holloway et al. 2014), so this issue, like all the rest discussed so far, remains to be resolved.

Humans are not the only primates to have large neonates relative to pelvic dimensions (Figure 1). This understanding, partnered with the increasing awareness of social behavior during nonhuman primate parturition, poses quite a challenge to human uniqueness. The first scientific observation of wild bonobo birth was recently published, and not only was it attended by interested group mates, but it added one more data point to the long list of similar observations published by primatologists since the 1960s (Douglas 2014).

Primate birth is a social event, regardless of fetal rotation or tightness of fit, which suggests that identifying the origins of these pelvic-based phenomena in hominin history is not a strong basis for reconstructing a shift in hominin social behavior. That is, we can assume that if ancient hominins were social primates, then they were true to form during parturition as well. A more active midwife-like assistance in childbirth performed uniquely by humans might require language to share and preserve that knowledge or a heightened cognitive capacity to create that knowledge in the first place. Such culture is not usually hypothesized to extend any further back than the past two million years with the origin of the genus Homo (to be generous). Thus, it may or may not be a coincidence that at least one of the contributors to difficult childbirth, marked encephalization, arose during the evolution of the genus Homo, but it will be difficult to demonstrate the point at which a midwife's knowledge and/or assistance would have become necessary (Rosenberg \& Trevathan 1995). Even with a more complete fossil record, it is not easy to imagine how to reconstruct whether and how extinct hominins assisted one another in childbirth beyond the social support that might be universal among anthropoid primates.

Although we do not have direct evidence for the origins or evolution of birthing relatively large babies, it is easy to argue that this phenomenon contributed to the tight fit. However, 
it is not as easy to argue why the human pelvis contributes, and it is even less easy to argue that it must. Taphonomy, combined with the unique anatomies displayed by extinct fossil taxa, and the increasing variation in the hominin pelvis displayed by each new addition to the record (e.g., Kibii et al. 2011) complicate matters. Looking to the fossil record to determine which evolving aspects of the pelvis are due to selection for bipedalism is not an easy task, nor is identifying when the tight fit at childbirth arose or when the corkscrew pattern of exit originated. The hominin pelvis evolved for numerous reasons over the past several million years, which makes it unlikely that present human anatomy is what is required for bipedalism, as it is so often phrased.

Pelvic evolution and present variation are explained by a combination of the following: adaptations to locomotion and to childbirth in an evolving body and evolving environment, sexual selection on an evolving body in an evolving environment, loss of tail, morphological integration, canalization, phylogenetic constraints, genetic drift, diet, behavior, climate, and other environmental influences during development or life (e.g., Abitol 1996, Lovejoy 2005, Dixson 2009, Ruff 2010, Grabowski et al. 2011, Lewton 2011, Tague 2011, Betti et al. 2013, Kurki 2013). But during hominin evolution, selection consistently favored adequately sized birth canals; that much is absolutely clear. Likewise, selection consistently favored neonates capable of fitting through those birth canals. So the few pelvic specimens in the hominin fossil record are testament to the variable morphology that has worked both for bipedalism as well as for childbirth over the past several million years. Of course, that is true only as long as each hominin pelvis on record did not end up there because of selection against its bipedal or birthing morphology. Fossils may be best for preserving hominin paleobiology, but they may be blinding us to other significant contributors to childbirth difficulty, including, but not limited to, position of the laboring woman; position of the fetus and the umbilical cord; function of the placenta, uterus, and cervix; muscular and bodily weakness; slow labor progression; multiple fetuses; preeclampsia; gestational diabetes; and young or old age at first birth.

The most recent and most thorough exploration of causes of childbirth difficulty through hominin evolutionary history was performed by Wells et al. (2012), and followed up by Wells 2015. They noted that although the tight fit between the fetus and the mother's pelvis may have occurred millions of years prior, agriculture has had a remarkable, and perhaps the most dramatic, effect. Agriculture has created opportunities for both malnourishment and over-nourishment, affecting both the growth of a mother's pelvis and the growth of her fetus during gestation. Undernourished mothers can birth relatively large babies owing to adaptive responses to protect fetal growth during pregnancy (Konje \& Ladipo 2000, Prentice \& Goldberg 2000), and regardless of maternal condition, larger babies are associated with longer labors and higher incidences of medical interventions (Turner et al. 1990). It is highly likely that there was never more childbirth difficulty than there is now and in recent history.

In sum, based on the limited pelvic remains in the fossil record at present, childbirth difficulty may have occurred as early as four million years ago in Australopithecus. But childbirth is a much more dynamic process than can be reconstructed from bones alone, so the hominin fossil record provides limited and tenuous information. Thus, it is difficult to explain childbirth difficulty with only the increase in body and brain size and the metamorphosis of the pelvis during hominin evolution. For now, the answer to the question of when childbirth became difficult is still soundly answered: when body and brain sizes approximated modern ones $<500,000$ years ago, or even more recently when agriculture and its consequences began to significantly impact human growth. Finally, documented social behavior associated with nonhuman primate births weakens our ability to infer uniquely human social behavior at birth based on hominin pelvic morphology. 


\section{DOES DIFFICULT CHILDBIRTH HAVE SOMETHING TO DO WITH INFANT HELPLESSNESS?}

The OD hypothesis also explains infant helplessness: Seemingly premature human neonates that require highly invested parenting are consequences of, or solutions to, the tight fit or the dilemma caused by competing selection for encephalized neonates and bipedal pelves. However, the accumulation of relevant evidence over the years has weakened the OD as an explanation for helpless human offspring. That is, intense childbirth difficulty and intensive parenting are not so easily explained by bipedal pelvic anatomy. Gestation length, fetal growth, childbirth processes, and neonatal helplessness are all connected to the bipedal hominin pelvis, but whether they are fundamentally influenced by it is not an easy argument to make.

A core issue here is whether humans are born early or receive less investment in utero (Dunsworth et al. 2012). However, human gestation is not short and seems to be even longer than expected for a primate of its body size. Furthermore, a human mother does not invest less in pregnancy than expected; she bears a large infant with a large infant brain for a primate of her body size. It is only when neonatal brain size is compared with adult brain size that human infants appear to be born early for primates.

Humans grow relatively less of their total brain size in utero, being born with only about $30 \%$ of their adult brain size and having to achieve more postnatal brain growth than any other primate (DeSilva \& Lesnik 2006). Chimpanzees grow relatively more of their full brain size than humans do by the time they are born, but they still get only $40 \%$ of the way there. If humans are removed from the comparison, chimpanzees appear to be born early compared with other primates such as capuchins, which are born with $50 \%$ of their adult brain size (Fragaszy et al. 2004).

So what has driven the tendency to label humans as altricial for so long is, from the infant's perspective, the fact that they are born with relatively small brains. That is, humans would need to gestate about seven more months to be born with the same proportion of brain growth accomplished that a chimpanzee has at birth (Portmann 1969, Gould 1977, Leutenegger 1982, DeSilva \& Lesnik 2006). This perspective assumes that brain size relative to adulthood is important for the timing of birth. Thus it appears that brain growth in utero is truncated, and this is due either to the OD or to Portmann's "extrauterine spring" hypothesis (1969); the latter proposes that infants are born early to experience enriching stimulation outside the womb while their brains develop.

These are the two different perspectives on human gestation length: from the infant brain's (OD) and from the mother's. The former suggests a unique end to gestation in humans, timing parturition to occur prior to the brain's lethal enlargement relative to the birth canal. However, the latter, from the mother's perspective, suggests a metabolic and energetic end to gestation because human neonates and their brains are as large or larger than expected for maternal body size and gestation is as long or longer than expected.

From this maternal perspective, the birth canal coevolved with maternal metabolism to adequately accommodate the upper limit on fetal size that the mother's physiology can tolerate during pregnancy, which in humans is relatively large. We call this hypothesis the energetics of gestation and fetal growth (EGG; Dunsworth et al. 2012) (Figure 2). Although the data are far better for humans, the hypothesis may also apply to other species because, among placental mammals, maternal body size (a proxy for metabolic parameters) is a good predictor of gestation length, fetal mass, and fetal brain mass (e.g., Sacher \& Staffeldt 1974; Martin 1983, 1996, 1998).

From the EGG perspective, gestation ends and the birth process is initiated when pregnancy reaches a critical point at which the mother can no longer support her growing fetus. The hormonal cascade that is involved in triggering birth has been described by Ellison's metabolic crossover hypothesis (2001; see also Dunsworth et al. 2012). From this metabolic and energetic perspective, 


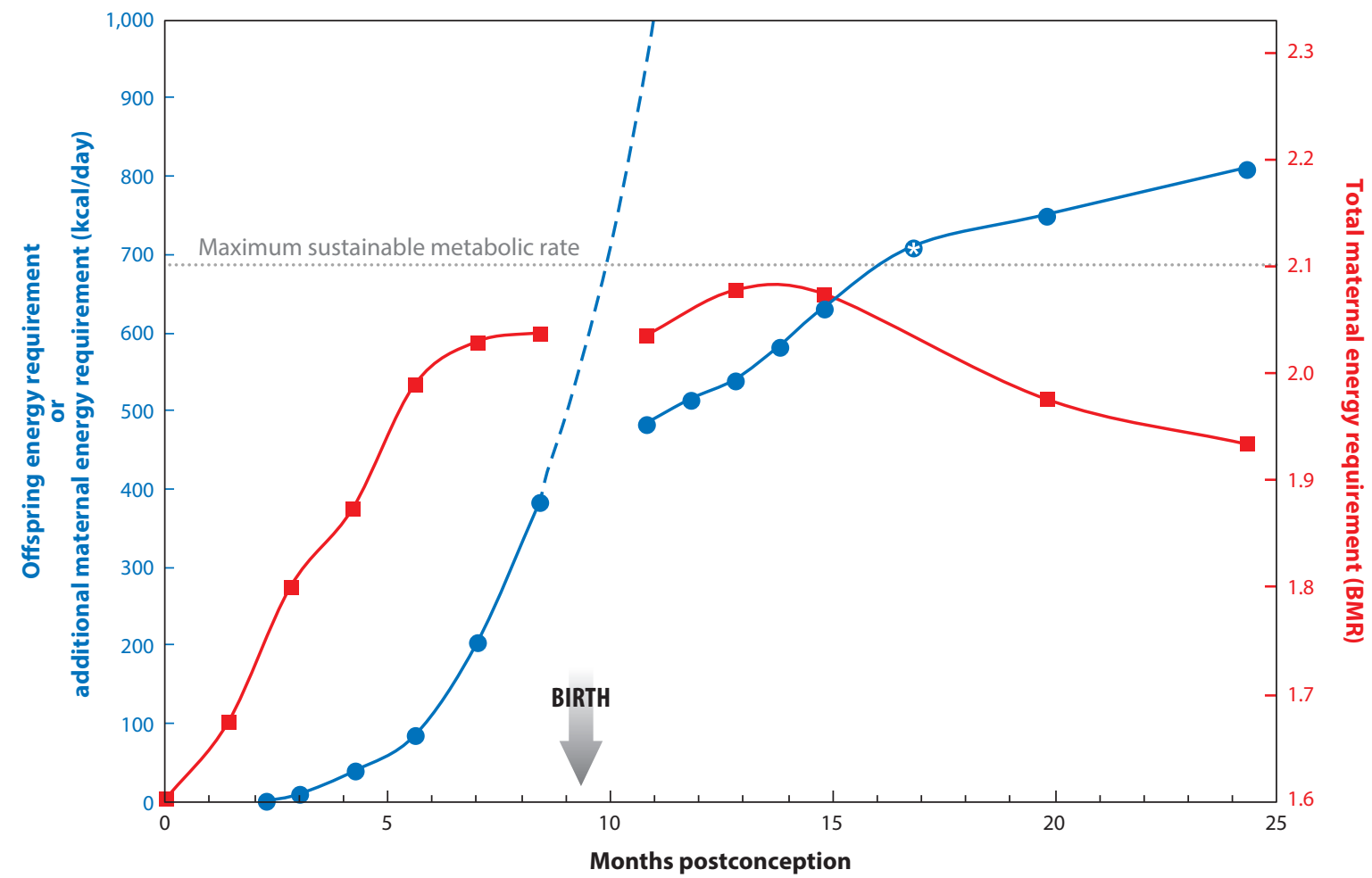

Figure 2

Metabolic constraint on gestation length and fetal size. Fetal energy demands (blue circles) increase exponentially during gestation. Maternal energy expenditure (red squares) rises during the first two trimesters but reaches a metabolic ceiling in the third, as total energy requirements approach $2.0 \times$ basal metabolic rate (BMR). Projected fetal energy requirements for growth beyond nine months (blue dashed line) quickly exceed the maximum sustainable metabolic rates for human mothers (horizontal, dotted line). After parturition (arrow), infant energy demands increase more slowly, and maternal energy requirements do not exceed $2.1 \times \mathrm{BMR}$. Required maternal energy expenditure for a fetus developmentally similar to a chimpanzee newborn (7-month-old infant; blue circle with white asterisk) would entail maternal energy requirements greater than $2.1 \times$ BMR. In humans, maximum sustained metabolic rate is thought to be 2.0-2.5 × BMR (Peterson et al. 1990, Hammond \& Diamond 1997). Adapted with permission from Dunsworth et al. (2012), which includes supporting references for the metabolic theory as well as for the data plotted in the figure.

difficult childbirth has not caused a specific end to human gestation or required that humans are born underdeveloped relative to other primates.

Hypotheses that humans are born too soon derive from classic comparisons of newborn humans to other species. Mammals fall along a spectrum from precocial (relatively more developed) to altricial (relatively less developed) in their anatomy and behavior at birth and through infancy. Primates as an order align with the precocial mammals, but humans have been singled out as "secondarily altricial" (Portmann 1969, Gould 1977), despite being unlike altricial animals in many ways (Robson et al. 2006, Trevathan \& Rosenberg in press).

As knowledge has increased over the past several decades, it has become increasingly difficult to single humans out as altricial mammals or altricial primates. As mentioned above, the human brain at birth is absolutely larger than any other primate's at birth. But unlike altricial mammals, human eyes and ears are open at birth and relative human furlessness is permanent. Like altricial mammals, humans can hardly move about independently, but neither do newborn apes (Douglas 2014). Human locomotion appears to be delayed but objectively might not be: When body size 
and foot position are taken into account, humans begin walking just when expected for a mammal (Garwicz et al. 2009).

Perhaps the most relevant difference between newborn humans and apes is that infant apes are eventually able to cling to their mothers and infant humans are not, at least not as early in development. Many factors contribute to human infants' clinging deficits. Perhaps skeletal immaturity plays a role (Watts 1990), as might the fact that humans produce large heavy babies that have relatively weak muscles. A relatively large head probably slows a human infant's motor behavior as well. Prioritization of brain development over muscle development could help explain human infant behavioral immaturity, as could heterochrony of the development of regional musculature (Grand 1992, Walker 2009). Certainly the loss of grasping feet and toes during the early hominin evolution - by the time australopiths made footprints at Laetoli at 3.6 million years ago-contributes to human infants' inability to cling as well. Other contributors to so-called helplessness can be blamed on adults rather than on infants. Perhaps, for primates of their body size and strength, human infants are a significant burden for human caregivers to carry (WallScheffler et al. 2007). Variation in parental behavior also contributes to the timing of infant motor skill development; humans living in WEIRD (Western, educated, industrialized, rich, democratic) populations have been associated with delayed onset of infant sitting and walking (Kasarik et al. 2010). Surely observations restricted to WEIRD populations are biasing how we perceive infant human development in an evolutionary framework.

In sum, little about human pregnancy, gestation, and fetal growth suggests that infants are born early or too soon, which suggests that difficult childbirth due to locomotor constraint on the bony birth canal is not a strong explanation for the timing of birth or infant helplessness. Humans appear to reach a metabolic capacity around nine months of gestation, and combined fetal-maternal metabolic stress is a solid hypothesis for that initiates the birth process. It is not clear whether it will be possible to demonstrate that the bipedal pelvis has truncated gestation or fetal growth, resulting in the birth of underdeveloped neonates. Factors that contribute to childbirth difficulty — such as overall size and adiposity of the infant, and muscular weakness and bipedalism of the infant and the mother-may also contribute to infant helplessness. Their inability to cling combined with their large size and the species' deficit in muscular strength, in both infants and caregivers, help to make human offspring a burden that is mitigated with cooperative breeding and culture. Whether helpless infants selected for cooperative breeding and culture, or whether the latter allowed for the former, is likely to remain a puzzle for those attempting to untangle these phenomena.

\section{EXPLORING FURTHER}

Not only is the human birthing process long and painful, but it is burdened with an injury and mortality risk to the fetus and the mother that is exceptional among primates. Moreover, few women bear offspring unattended or unassisted. What the mother and fetus experience and the cultural nature of it are both defining traits of the human species. With enough evidence, we should be able to determine whether the differences we observe in humans can be attributed to their unique adaptation and whether the species' brand of reproduction can be evolutionarily linked to other traits that arose in the lineage such as bipedal locomotion, encephalization, intense prosociality, heightened cognition, and culture. Reconstructing the evolution of childbirth, both for the sake of knowledge but also for the potentially positive impacts on present childbirth practices and outcomes, makes it an important enterprise despite the numerous questions that remain and the impressive workload that still needs to be accomplished. 
With the discovery of each new fossil hominin pelvis, or with each new, alternative pelvic reconstruction, there will be new light shed on the evolution of childbirth. With greater understanding of the link between anatomical variation and function, paleoanthropologists will better be able to tease apart pelvic adaptations for childbirth that might be compromised by those for bipedalism and vice versa, in both living and extinct hominins. This work will be easier as sexual dimorphism, particularly in the pelvis, is better understood through hominin history (Plavcan 2000). Functional interpretations of the hominin pelvis will also be easier as the model for the last common ancestor between humans and chimpanzees is better understood. Perhaps we have been downplaying monkeylike hominin ancestral anatomy and for both living and fossil apes and monkeys will continue to improve the human anatomical and behavioral models for the earliest hominins.

Furthermore, with an increased realization among paleoanthropologists that females are the gateway to continued evolution (Wall-Scheffler 2012), it is possible that the pregnant and lactating hominin female will garner more intense and creative scientific study by paleoanthropologists and other scientists. Human skeletal (and other) traits in females, and even in males, are likely the product of natural selection on the childbearing and -rearing female. Although it is exaggerated in women, the lumbar lordosis may be one such trait (Whitcome et al. 2007). Perhaps the pelvis is limited or altered in certain dimensions not for bipedal walking and running for all hominins, but because of selection for the locomotion of pregnant females or females carrying infants (WallScheffler et al. 2007). Perhaps sexual dimorphism is diminished in humans not because males have decreased in size but because females have increased. Bearing and rearing large infants might not have evolved if hominin females were appreciably smaller than males. There is clearly still much to be gained from studying and augmenting the hominin fossil record; however, other types of research potentially hold greater promise for answering interesting questions about the evolution of childbirth.

There is still much to learn about pregnancy, parturition, infancy, and infant-rearing in nonhuman primates. Nonhuman primates hold many, if not most, of the answers to our questions about what makes human childbirth comparatively difficult and what makes human infants (and parents) comparatively helpless. Data from behavioral observations will be crucial, including data on pregnant female primate locomotion, sociality, diet, caloric intake, fat deposition, weight gain, oxygen consumption, and sleep behavior, as well the equivalent data on infants and lactating females and alloparents. Metabolic, endocrinological, and other physiological evidence for all these phases and events in life will be increasingly important, as will intensive anatomical study of fetal and infant ontogeny, especially when linked to the behavioral and physiological data. Increased understanding of behavior, especially positional and social behavior, during labor and parturition will be insightful. Pregnancy and lactation energetics will be keys to understanding whether a species is gestating and nursing to a metabolic and/or energetic maximum or whether it is altering gestation and fetal growth and lactation compared with predictions.

Without increasing our ability to make primate comparisons, there is little hope for knowing how bipedalism, adiposity, taillessness, encephalization, culture, etc. contribute to the evolution of pregnancy, childbirth, infancy, and how we parent and alloparent. The primate studies (and those of all placental mammals relevant for answering these questions), especially those focused on metabolism and behavior (Pontzer et al. 2010, Thompson 2013, Pontzer et al. 2014), are crucial if we are to explain how humans have such costly infants yet have much shorter interbirth intervals than do the rest of the hominoids. Without more comparison we cannot know how costly human pregnancies and the resulting infants truly are, and for example, we cannot understand the role of the placenta and how it varies in relation to other traits among species. Primates that regularly birth 
twins and practice cooperative breeding, such as marmosets, hold great potential for answering many questions about reproductive physiology, metabolic limits, and behavioral correlates (e.g., Rutherford \& Tardiff 2008).

Published data such as gestation length may appear to be straightforward but, in fact, are not (Borries et al. 2013). And as more and better gestation data accumulate, especially for apes (e.g., Wildman et al. 2011), it may be possible to reconsider whether human gestation is comparatively short, or for example, if species that lack restrictive bony birth canals [cetaceans and sirenians (manatees and dugongs)] have comparatively long gestations. If they do, then humans would not be unique in having a life history altered by the pelvis, and therefore the OD might not seem so unlikely despite the metabolic hypothesis.

The trigger or, perhaps, multiple triggers that initiate human parturition remain unknown (e.g., Newman et al. 2014). This seems like an important area for intense research focus both on humans and on their closest primate relatives. The clinical implications are, of course, significant, but such knowledge would also shed light on why chimpanzees and many other primates and mammals give birth so far in advance of reaching a pelvic limit.

How do we use any of this evolutionary perspective to inform current childbirth practices? Attempting to answer this question will breach the bounds of this article (see Liston 2003, Roy 2003, Pike 2005, Rosenberg \& Trevathan 2007, Wittman \& Wall 2007, Davis-Floyd \& Cheyney 2009, Brown et al. 2013, Basso 2014, Newman et al. 2014). However, the OD is very much a part of the present zeitgeist; it factors into how parents are advised to parent their seemingly altricial infants and how women elect to schedule cesarean sections out of fear of childbirth. The typological thinking applied to fossil hominin pelvic analyses and favored in an OD framework may be limiting present appreciation for the normal variation in pelvic anatomy and the birthing process that has been tolerated by natural selection (Walrath 2003). Furthermore, medical professionals might believe that cesarean sections are an evolutionary imperative (Weiner et al. 2008) rather than a cultural one, which may lead some practitioners to perform interventions when they are unnecessary. Judging whether interventions are necessary, however, is far from easy, especially while armed with the culture and resources to reduce mortality risks to mothers and infants. In addition, larger babies seem to exhibit better postnatal cognitive outcomes (Figlio et al. 2014), so pregnancies that test the limits of the pelvis, and births that occur in operating rooms, may exemplify a different kind of reproductive success than hominins knew for most of evolutionary history. Childbirth in this present chapter of hominin evolutionary history should prove to be a wonderland for anthropologists. Answering questions directly and indirectly related to the evolution of childbirth may never satiate our quest for specific details about hominin prehistory. But the answers will shed light on how humans have come to dominate the planet in spite of the difficulty we face in bearing infants.

\section{DISCLOSURE STATEMENT}

The authors are not aware of any affiliations, memberships, funding, or financial holdings that might be perceived as affecting the objectivity of this review.

\section{ACKNOWLEDGMENTS}

Samantha Tickey, Sharon DeWitte, Pat Shipman, Jeremy DeSilva, Karen Rosenberg, and Wenda Trevathan provided helpful discussion and insight while we were writing this article. For that, and for the researchers who performed the work we discussed here, we are grateful. 


\section{LITERATURE CITED}

Abitol MM. 1987. Obstetrics and posture in pelvic anatomy. F. Hum. Evol. 16(3):243-55

Abitol MM. 1993. Adjustment of the fetal head and adult pelvis in modern humans. Hum. Evol. 8(3):167-85

Abitol MM. 1996. The shapes of the female pelvis. Contributing factors. F. Reprod. Med. 41(4):242-50

Aiello LC, Wells JCK. 2002. Energetics and the evolution of the genus Homo. Annu. Rev. Anthropol. 31:323-38

Arsuaga J-L, Lorenzo C, Carretero J-M, Gracia A, Martínez I, et al. 1999. A complete human pelvis from the Middle Pleistocene of Spain. Nature 399:255-58

Basso O. 2014. Reproductive epidemiology in an evolutionary perspective: why bigger may not be better. Curr. Epidemiol. Rep. 1:98-101

Berge C, Goularas D. 2010. A new reconstruction of Sts 14 pelvis (Australopithecus africanus) from computed tomography and three-dimensional modeling techniques. 7. Hum. Evol. 58:262-72

Berge C, Orban-Segebarth R, Schmid P. 1984. Obstetrical interpretation of the Australopithecine pelvic cavity. F. Hum. Evol. 13:573-87

Betti L, von Cramon-Taubadel N, Manica A, Lycett SJ. 2013. Global geometric morphometric analyses of the human pelvis reveal substantial neutral population history effects, even across sexes. PLOS ONE 8(2):e55909

Borries C, Gordon AD, Koenig A. 2013. Beware of primate life history data: a plea for data standards and a repository. PLOS ONE 8(6):e67200

Brown EA, Ruvolo M, Sabeti PC. 2013. Many ways to die, one way to arrive: how selection acts through pregnancy. Trends Genet. 29(10):585-92

Chene G, Tardieu AS, Trombert B, Amouzougan A, Lamblin G, et al. 2014. A species' odyssey: evolution of obstetrical mechanics from Australopithecus Lucy to nowadays. Euro 7. Obstet. Gynecol. Reprod. Bio. 181:316-20

Clutton-Brock T. 2002. Breeding together: kin selection and mutualism in cooperative vertebrates. Science 296(5565):69-72

Cunnane SC, Crawford MA. 2003. Survival of the fattest: Fat babies were the key to evolution of the large human brain. Comp. Biochem. Phys. Part A 136:17-26

Davis-Floyd R, Cheyney M. 2009. Birth and the big bad wolf: an evolutionary perspective. In Childbirth Across Cultures: The History of Non-Western Science, ed. H Selin, PK Stone, pp. 1-23. New York: Springer

DeSilva J. 2011. A shift toward birthing relatively large infants early in human evolution. PNAS 108(3):1022-27

DeSilva J, Lesnik J. 2006. Chimpanzee neonatal brain size: implications for brain growth in Homo erectus. 7. Hum. Evol. 51:207-12

Dixson A. 2009. Sexual Selection and the Origins of Human Mating Systems. Oxford, UK: Oxford Univ. Press

Dolea C, AbouZahr C. 2003. Global burden of obstructed labor in the year 2000. In Global Burden of Disease 2000, pp. 1-17. Geneva: Evid. Inf. Policy, World Health Organ. http://www.who.int/healthinfo/ statistics/bod_obstructedlabour.pdf

Douglas PH. 2014. Female sociality during the daytime birth of a wild bonobo at Luikotale, Democratic Republic of the Congo. Primates 55:533-42

Dunsworth HM, Warrener AG, Deacon T, Ellison PT, Pontzer H. 2012. Metabolic hypothesis for human altriciality. PNAS 109(38):15212-16

Ellison P. 2001. On Fertile Ground: A Natural History of Human Reproduction. Cambridge, MA: Harvard Univ. Press

Falk D, Zollikofer CPE, Moromoto N, Ponce de Leon MS. 2012. Metopic suture of Taung (Australopithecus africanus) and its implications for hominin brain evolution. PNAS 109:8467-70

Figlio D, Guryan J, Karbownik K, Roth J. 2014. The effects of poor neonatal health on children's cognitive development. Work. Pap. Ser, Inst. Policy Res., Northwest. Univ.

Fragaszy DM, Visalberghi E, Fedigan LM. 2004. The Complete Capuchin: The Biology of the Genus Cebus. Cambridge, UK: Cambridge Univ. Press

Garwicz M, Christensson M, Psouni E. 2009. A unifying model for timing of walking onset in humans and other mammals. PNAS 106(51):21889-93

Gould SJ. 1977. Ontogeny and Phylogeny. Cambridge, MA: Harvard Univ. Press 
Grabowski MW, Polk JD, Roseman CC. 2011. Divergent patterns of integration and reduced constraint in the human hip and the origins of bipedalism. Evolution 65:1336-56

Grand TI. 1992. Altricial and precocial mammals: a model of neural and muscular development. Zoo Biol. $11: 3-15$

Green D, Alemseged Z. 2012. Australopithecus afarensis scapular ontogeny, function, and the role of climbing in human evolution. Science 338:514-17

Hammond KA, Diamond J. 1997. Maximal sustained energy budgets in humans and animals. Nature 386:45762

Hirata S, Fuwa K, Sugama K, Kusunoki K, Takashita H. 2011. Mechanism of birth in chimpanzees: Humans are not unique among primates. Biol. Lett. 7(5):686-88

Holloway RL, Boadfied DC, Carlson KJ. 2014. New high-resolution computed tomography data of the Taung partial cranium and endocast and their bearing on metopism and hominin brain evolution. PNAS 111(36):13022-27

Hrdy S. 2009. Mothers and Others. Cambridge, MA: Harvard Univ. Press

Kasarik LB, Adolph KE, Tamis-Lemonda CS, Bornstein MH. 2010. WEIRD walking: cross-cultural research on motor development. Behav. Brain Sci. 33(2-3):95-96

Kibii JM, Churchill SE, Schmid P, Carlson KJ, Reed ND, et al. 2011. A partial pelvis of Australopithecus sediba. Science 333:1407-11

Konje JC, Ladipo OA. 2000. Nutrition and obstructed labor. Am. F. Clin. Nutr. 72:291-97

Kramer KL. 2005. Children's help and the pace of reproduction: Cooperative breeding in humans. Evol. Anthropol. 14(6):224-37

Krogman W. 1951. The scars of human evolution. Sci. Am. 185:54-57

Kurki HK. 2013. Skeletal variability in the pelvis and limb skeleton of humans: Does stabilizing selection limit female pelvic variation? Am. F. Hum. Biol. 25(6):795-802

Kuzawa CW. 1998. Adipose tissue in human infancy and childhood: an evolutionary perspective. Am. F. Phys. Anthropol. 107(Suppl. 27):177-209

LaVelle M. 1995. Natural selection and developmental sexual variation in the human pelvis. Am. F. Phys. Anthropol. 98:59-72

Leutenegger W. 1982. Encephalization and obstetrics in primates with particular reference to human evolution. In Primate Brain Evolution: Methods and Concepts, ed. E Armstrong, D Falk, pp. 85-95. New York: Plenum

Lewton KL. 2011. Evolvability of the primate pelvic girdle. Evol. Biol. 39:126-39

Liston WA. 2003. Rising caesarean section rates: Can evolution and ecology explain some of the difficulties of modern childbirth? 7. R. Soc. Med. 96:559-61

Lovejoy CO. 2005. The natural history of human gait and posture: Part 1. Spine and pelvis. Gait Posture 21:95-112

Lovejoy CO, Suwa G, Spurlock L, et al. 2009. The pelvis and femur of Ardipithecus ramidus: the emergence of upright walking. Science 326:71

Martin RD. 1983. Human Brain Evolution in an Ecological Context. Fifty-Second James Arthur Lect. Evol. Hum. Brain. New York: Am. Mus. Nat. Hist.

Martin RD. 1996. Scaling of the mammalian brain: the maternal energy hypothesis. News Pbysiol. Sci. 11:149-56

Martin RD. 1998. Comparative aspects of human brain evolution: scaling, energy costs and confounding variables. In The Origin and Diversification of Language, ed. NG Jablonski, LC Aiello, pp. pp. 35-68. San Francisco: Calif. Acad. Sci.

Newman T, Lengyel C, Pavlicev M, Muglia LJ. 2014. Human evolution, genomics and birth timing: new approaches for investigating preterm birth. NeoReviews 15:17

Peterson CC, Nagy KA, Diamond J. 1990. Sustained metabolic scope. PNAS 87:2324-28

Pike I. 2005. Maternal stress and fetal responses: evolutionary perspectives on preterm delivery. Am. F. Hum. Biol. 17:55-65

Plavcan M. 2000. Inferring social behavior from sexual dimorphism in the fossil record. 7. Hum. Evol. 39(3):327-44

Pontzer H, Raichlen DA, Gordon AD, Schroepfer-Walker KK, Hare B, et al. 2014. Primate energy expenditure and life history. PNAS 111(4):1433-37 
Pontzer H, Raichlen DA, Shumaker RW, Ocobock C, Wich SA. 2010. Metabolic adaptation for low energy throughput in orangutans. PNAS 107(32):14048-52

Portmann A. 1990 (1969). A Zoologist Looks at Humankind, transl. J Schaefer. New York: Columbia Univ. Press (from German)

Prentice AM, Goldberg GR. 2000. Energy adaptations in human pregnancy: limits and long-term consequences. Am. 7. Clin. Nutr. 71:1226-32

Roberts AM, Thorpe SKS. 2014. Challenges to human uniqueness: bipedalism, birth and brains. F. Zool. 292:281-89

Robson SL, van Schaik CP, Hawkes K. 2006. The derived features of human life history. In The Evolution of Human Life History, ed. K Hawkes, RR Paine, pp. 17-44. Oxford, UK: K. James Currey

Rosenberg K, Trevathan WR. 2002. Birth, obstetrics, and human evolution. B7OG 109(11):1199-206

Rosenberg K, Trevathan WR. 2007. An anthropological perspective on the evolutionary context of preeclampsia in humans. F. Reprod. Immunol. 76:91-97

Rosenberg KR. 1992. The evolution of modern human childbirth. Yearb. Phys. Antbropol. 35:89-124

Rosenberg KR, Trevathan WR. 1995. Bipedalism and human birth: the obstetrical dilemma revisited. Evol. Anthropol. 4(5):161-68

Rosenberg KR, Trevathan WR. 2014. Evolutionary obstetrics. Evol. Med. Public Health 2014:148

Roy RP. 2003. A Darwinian view of obstructed labor. Obstet. Gynecol. 101(2):397-401

Ruff CB. 2010. Body size and body shape in early hominins: implications of the Gona pelvis. 7. Hum. Evol. 58:166-78

Rutherford JN, Tardiff SD. 2008. Placental efficiency and intrauterine resource allocation strategies in the common marmoset pregnancy. Am. F. Phys. Anthropol. 137(1):60-68

Sacher GA, Staffeldt EF. 1974. Relation of gestation time to brain weight for placental mammals: implications for the theory of vertebrate growth. Am. Nat. 18:593-615

Schultz A. 1949. Sex differences in the pelves of primates. Am. 7. Phys. Anthropol. 7(3):401-24

Simpson SW, Quade J, Levin NE, Butler R, Dupont-Nivet G, et al. 2008. A female Homo erectus pelvis from Gona, Ethiopia. Science 322:1089-92

Tague RG. 2011. Fusion of coccyx to sacrum in humans: prevalence, correlates, and the effect on pelvic size, with obstetrical and evolutionary implications. Am. F. Phys. Anthropol. 145:426-37

Tague RG, Lovejoy CO. 1986. The obstetric pelvis of A. L. 288-1 (Lucy). 7. Hum. Evol. 15:237-94

Thompson ME. 2013. Comparative reproductive energetics of human and nonhman primates. Annu. Rev. Anthropol. 42:287-304

Trevathan W, Rosenberg K, eds. In press. Costly and Cute: How Helpless Babies Make Us Human. Santa Fe, NM: Sch. Am. Res.

Trevathan WR. 2015. Primate pelvic anatomy and implications for birth. Phil. Trans. Soc. B 370:20140065

Trevathan WR, Rosenberg K. 2000. The shoulders follow the head: postcranial constraints on human childbirth. F. Hum. Evol. 39:583-86

Trinkaus E. 1984. Neanderthal pubic morphology and gestation length. Curr. Antbropol. 25(4):509-14

Turner MJ, Rasmussen MJ, Boylan PC, MacDonald D, Stronge JM. 1990. The influence of birth weight on labor in nulliparas. Obstet. Gynecol. 76(2):159-63

Walker A. 2009. The strength of great apes and the speed of humans. Curr. Anthropol. 50(2):229-34

Wall-Scheffler CM. 2012. Energetics, locomotion, and female reproduction: implications for human evolution. Annu. Rev. Anthropol. 41:71-85

Wall-Scheffler CM, Geiger K, Steudel-Number KL. 2007. Infant carrying: the role of increased locomotory costs in early tool development. Am. 7. Phys. Anthropol. 133:841-46

Walrath D. 2003. Rethinking pelvic typologies and the human birth mechanism. Curr. Anthropol. 44:5-31

Warrener A, Lewton K, Pontzer H, Lieberman D. 2015. A wider pelvis does not increase locomotor cost in humans, with implications for the evolution of childbirth. PLOS ONE 10(3):e0118903

Washburn S. 1960. Tools and human evolution. Sci. Am. 203:3-15

Watts E. 1990. Evolutionary trends in primate growth and development. In Primate Life History and Evolution, ed. C DeRousseau, pp. 89-104. New York: Wiley-Liss

Weaver TD, Hublin J-J. 2009. Neandertal birth canal shape and the evolution of human childbirth. PNAS 106(20):8151-56 
Weiner S, Monge J, Mann A. 2008. Bipedalism and parturition: an evolutionary imperative for Cesarean delivery? Clin. Perinatol. 35(3):469-78

Wells JCK. 2015. Between Scylla and Charybdis: renegotiating resolution of the 'obstetric dilemma' in response to ecological change. Philos. Trans. R. Soc. B 370:20140067

Wells JCK, Desvar Stock JT. 2012. The obstetric dilemma: an ancient game of Russian roulette, or a variable dilenmma sensitive to ecology? Yearb. Phys. Anthropol. 149(55):40-71

WHO (World Health Organ.). 2005. World Health Report: Make Every Woman and Child Count. Geneva: WHO

Whitcome KK, Shapiro LJ, Lieberman DE. 2007. Fetal load and the evolution of lumbar lordosis in bipedal hominins. Nature 450:1075-78

Wildman DE, Uddin M, Romero R, Gonzalez JM, Than NG, et al. 2011. Spontaneous abortion and preterm labor and delivery in nonhuman primates: evidence from a captive colony of chimpanzees (Pan troglodytes). PLOS ONE 6(9):e24509

Wittman AB, Wall LL. 2007. The evolutionary origins of obstructed labor: bipedalism, encephalization, and the human obstetric dilemma. Obstet. Gynecol. Surv. 62:739-48 